\title{
A Beam Broadening Method for Phased Arrays in Wireless Communications
}

Corentin Fonteneau ( $\nabla$ corentin.fonteneau@insa-rennes.fr)

Orange SA https://orcid.org/0000-0003-2197-9214

Matthieu Crussière

IETR

Bruno Jahan

Groupe Orange: Orange SA

\section{Research}

Keywords: Analog beamforming, phase array, millimeterwaves, 5G

Posted Date: November 1st, 2021

DOI: https://doi.org/10.21203/rs.3.rs-980108/v1

License: (c) (1) This work is licensed under a Creative Commons Attribution 4.0 International License.

Read Full License 


\title{
A Beam Broadening Method for Phased Arrays in Wireless Communications
}

\author{
Corentin Fonteneau $^{\dagger \ddagger}$, Matthieu Crussière ${ }^{\ddagger}$, Bruno Jahan ${ }^{\dagger}$ \\ †Orange Labs, Rennes, France, corentin.fonteneau@ orange.com \\ ${ }^{\ddagger}$ Univ Rennes, INSA Rennes, IETR - UMR 6164 F-35000 Rennes, France
}

\begin{abstract}
G and IEEE 802.11ay introduce the use of the millimeter band as one promising solution to provide broadband wireless communication at multi-Gb/s user data rate. Due to the severe path-loss at such frequencies, it is generally assumed that large antenna arrays are used at the base station to steer narrow beams and build highly directionnal communication links towards the terminal points. However, broader and less directional beams are also of high interest in some of the steps involved in the establishment or the maintenance of the communication links. Indeed, search of a large area by narrow beams becomes too time consuming and link outage becomes more critical, thus affecting the latency and the robustness of the communications. A method enabling an adaptation of the beam widths is then worthwhile to consider.

In this article, we investigate how narrow beams naturally produced by large antenna arrays can be broadened to adapt the beam width to a desired angular sector. We consider that the multi-antenna processing is performed by phase shifters on the radio-frequency stage since its digital counterpart is hardly feasible in practice at such high frequencies. The main idea of our systematic phase-only beam broadening technique relies on the determination of a quadratic phase excitation law from a desired beam width and steering angle. We first lead a thorough analysis of the radiation behavior regarding the coefficients of such quadratic excitation. We then propose a calculation method for determining the polynomial coefficients as a function of the desired beam width and steering angle. This non-iterative beam broadening method is described for boresight and non-boresight directions and is intended for discrete antenna arrays.
\end{abstract}

Index Terms-Analog beamforming, phase array, millimeterwaves, $\mathbf{5 G}$

\section{INTRODUCTION}

The recent introduction of the millimeter band in the last versions of the prevailing wireless communication standards, namely 5G NR and IEEE 802.11ay, is considered as one major enabler for the enhancement of the capacity of wireless networks. Working with carrier frequencies of several tens of $\mathrm{GHz}$ is indeed highly attractive owing to the very large bandwidth available in this portion of the radio spectrum. However, millimeter waves suffer from much higher propagation losses compared to lower frequencies. In addition to the strong path loss given by the well-known Friis transmission equation, signals at millimeter wave penetrate less easily through buildings, solid materials or even human bodies [1][3]. A convenient way to combat such drawbacks is to establish directional communications towards users or terminals by means of adaptive beamforming techniques.

Forming directional beams can for instance be easily implemented using a linear antenna array controlled by a linear phase excitation. The $3 \mathrm{~dB}$ beam width obtained in this way has the remarkable property of being inversely proportional to the antenna array length while the maximum gain is proportional to $10 \log _{10}(M) \mathrm{dB}$ where $M$ denotes the number of antennas composing the array [4]. Although those properties are theoretically beneficial in order to combat strong pathlosses and increase the received power, beam misalignment may occur in practical scenarios, especially with large antenna arrays, thus leading to poor link quality [5]. On one other hand, very narrow beams are costly regarding beam scanning latency time and not well-suited for broadcast channels that have to be received by several users [6], [7]. Finally, in regard to the penetration problems of millimeter waves, it has been shown that less-directional beams can improve link resilience since the energy from non-line-of-sight paths is retrieved [8], [9]. As depicted throughout these scenarios, being able to adapt and increase the width of the formed beams becomes essential at various levels of the communication link management.

Beam broadening techniques have always been a subject of research for radar applications [10], [11] and has more recently become a topic of interest for mobile wireless communications as the community started looking at the millimeter band. Generally speaking, a broadened beam can be designed and controlled using adequate amplitude and phase excitations [12]. Such an approach is well suited to fully digital beamforming implementation which is however hardly applicable to the millimeter wave context [13], [14]. Pure analog or at least hybrid analog-digital beamforming architectures have rather to be considered for millimeter wave front-ends [13], [15]. This implies that beam direction and width have to be managed at the analog stage. On that basis, amplitude excitation may be achieved by controlling the gains of the power amplifiers while phase excitation may be obtained through phase shifters. For power efficiency reasons, it is however recommended for millimeter wave applications that power amplifiers operate at maximum power rather than tuning their gains [16], [17]. Consequently, phase-only element weights are preferable in practical millimeter wave beamformers. Finding the phase excitation that ensures a given beam width then becomes a non linear and non convex optimization problem [18].

Optimization of beam broadening techniques constrained to unit amplitude weights have already been studied in many papers [10], [18], [19]. The obtained methods yield interesting results but lack of flexibility since the optimization process has to be done offline regarding particular predefined configura- 
tions. Contrary to these methods, authors in [17] provide a systematic approach for beam broadening. The idea consists in dividing the array into multiple logical subarrays, each being controlled by an independent linear phase excitation and being responsible for an elementary beam associated with a predefined direction. The resulting broadened beam is obtained by summation of the elementary ones. The major drawback of this method is that the number of broadened beams that can be formed is limited by the number of antenna elements comprised in the array. Indeed, each subarray has to be composed of the same number of antennas with the constraint that the number of subarrays should not exceed the number of antennas per subarray. For example, only three different beams are configurable for a 128-element antenna array. Authors in [20] proposed a similar approach for which the steering directions of the elementary beams are refined through an optimization process. This solution appears to be more flexible but also more computationally expensive. Other beam broadening strategies can finally be found as in [21], where the broadened beam patterns are obtained through nonlinear parametric phase excitations. However, no other method than an exhaustive search is suggested for tuning the phase law parameters regarding the desired beam width. Besides, such an approach can be quite tricky since different quadratic coefficients give the same beam width.

In this paper, we introduce a beam broadening control method that is based on a quadratic phase excitation and that involves two parameters. Our method requires neither an exhaustive search nor an iterative one to find the adequate control parameters. Throughout the article, we establish a bijective function linking these two parameters with the beam width and the steering angle of the power pattern produced by the phased array. To that purpose, an analysis of the farfield radiation pattern of the array is first led to identify how the proposed quadratic phase law governs the beam shape. It is then demonstrated that the coefficients of the quadratic phase excitation can be expressed according to a new variable that has a near linear relationship with the beam width, for boresight and non-boresight directions. In this work, the beam width is characterized from the beam power efficiency. This choice has been made since the conventional half power beam width is not relevant in some configurations for which the ripples can exceed $3 \mathrm{~dB}$. On that basis, we design our proposed beam broadening control method which can be implemented for various antenna array sizes, boresight and non-boresight directions.

The rest of the paper is organized as follows. In Section II some fundamental expressions about line-source radiation are reminded and the far-field radiation pattern for a quadratic phase excitation is derived. In Section III, a beam control method that relies on the Fresnel functions is derived for that type of excitation. The beam width definition is then given in Section IV and the relation between the Fresnel functions and the beam width is established for boresight and non-boresight directions. Finally, the accuracy of the proposed beam width control method is evaluated in Section $\mathrm{V}$ before concluding our work in Section VI.

\section{FAR-FIELD BEAM PATTERN OF LINE-SOURCES}

In this section, the general principles yielding the farfield radiation of a continuous line source are reminded and specifically derived and analyzed in case of a quadratic phase excitation. The obtained expressions serve as basis to the beam width control method developed in the sequel of the paper.

\section{A. Radiation pattern of a continuous line-source}

Following the conventional definition of the spherical coordinate system, the space factor $S F(\theta)$ for a continuous linesource of length $L$ placed symmetrically along the z-axis is given by [12],

$$
S F(\theta)=\int_{\frac{-L}{2}}^{\frac{+L}{2}} I(z) e^{\jmath\left(k_{0} z \cos \theta+\Phi(z)\right)} d z,
$$

where $\theta$ is the elevation angle, $k_{0}=\frac{2 \pi}{\lambda}$ represents the wave number with $\lambda$ the wavelength, while $I(z)$ and $\Phi(z)$ correspond respectively to the amplitude and phase distributions along the source. Following the common assumption that the source operates at maximum power rate without any amplitude change at millimeter wave frequencies, a uniform amplitude distribution is considered, i.e. $I(z)=\frac{I_{0}}{L}$. It is then noticeable that Eq. (1) relates the far-field pattern of the source to its excitation distribution through the Fourier transform of a complex exponential function $f(z)$ as,

$$
S F(\xi)=\frac{I_{0}}{L} \int_{\frac{-L}{2}}^{\frac{+L}{2}} f(z) e^{\jmath 2 \pi \xi z} d z
$$

with,

$$
f(z)=e^{\jmath \Phi(z)}, \quad \xi=\frac{1}{\lambda} \cos \theta .
$$

A classic excitation of the form $e^{j \Phi(z)}$ is the linear phase distribution defined as,

$$
\Phi(z)=B_{1} z,
$$

with,

$$
B_{1}=-k_{0} \cos \left(\theta_{\max }\right) .
$$

Such linear phase excitation has the interesting property of steering the maximum radiation towards the direction $\theta_{\max }$. One of the main drawback of this method is that the $3 \mathrm{~dB}$ beam width is not tunable for a given direction. Indeed, the half power beam width is inversely proportional to the source length $L$ and to $\sin \left(\theta_{\max }\right)$.

Since the far-field pattern is related to its excitation through the fourier transform (2), temporal waveforms of the form $e^{j \Phi(t)}$ with easily tunable power spreading properties in the frequency domain are good candidates for beam widening. The linear chirp is one of them as a configurable bandwidth can be swept by introducing quadratic variations on the instantaneous phase [22]. We thus propose to conduct a deeper study on the influence of a quadratic phase excitation on the far-field radiation pattern. 


\section{B. Radiation pattern with a quadratic phase excitation}

Let us consider a quadratic phase distibution of the form,

$$
\Phi(z)=B_{1} z+B_{2} z^{2},
$$

in which $B_{1}$ given by Eq. (5) is the coefficient that controls the linear phase shift between antenna elements, i.e the beam direction, while $B_{2}$ adds a quadratic phase shift that is expected to generate a broadened beam owing to the known spectrum shape of chirp signals mentioned above. Note that no constant coefficient is considered in the quadratic law since it would not affect the beam pattern. By integrating Eq. (6) into Eq. (1), we obtain

$$
S F(\theta)=\frac{I_{0}}{L} \int_{\frac{-L}{2}}^{\frac{+L}{2}} e^{j \phi(z)} d z
$$

with the vertex form of $\phi(z)$ being,

$$
\phi(z)=B_{2}\left(z+\frac{B_{1}+k_{0} \cos (\theta)}{2 B_{2}}\right)^{2}-\frac{\left(B_{1}+k_{0} \cos (\theta)\right)^{2}}{4 B_{2}} .
$$

Substituting variable $z$ by $u=\sqrt{\frac{2 B_{2}}{\pi}}\left(z+\frac{B_{1}+k_{0} \cos (\theta)}{2 B_{2}}\right)$, we get

$$
S F(\theta)=\frac{I_{0}}{L} \sqrt{\frac{\pi}{2 B_{2}}} e^{-\jmath \frac{\left(B_{1}+k_{0} \cos (\theta)\right)^{2}}{4 B_{2}}} I(\theta),
$$

where,

$$
\begin{aligned}
& I(\theta)=\int_{\underline{u}_{\theta}}^{\bar{u}_{\theta}} e^{j \frac{\pi}{2} u^{2}} d u, \\
& \text { with, }\left\{\begin{array}{l}
\underline{u}_{\theta}=\sqrt{\frac{2 B_{2}}{\pi}}\left(-\frac{L}{2}+\frac{B_{1}+k_{0} \cos (\theta)}{2 B_{2}}\right) \\
\bar{u}_{\theta}=\sqrt{\frac{2 B_{2}}{\pi}}\left(\frac{L}{2}+\frac{B_{1}+k_{0} \cos (\theta)}{2 B_{2}}\right)
\end{array} .\right.
\end{aligned}
$$

Eq. (9) can be rewritten using the normalized cosine Fresnel integral $C(u)=\int_{0}^{u} \cos \left(\frac{\pi}{2} t^{2}\right) d t$ and the normalized sine Fresnel integral $S(u)=\int_{0}^{u} \sin \left(\frac{\pi}{2} t^{2}\right) d t$ as [23],

$$
I(\theta)=C\left(\bar{u}_{\theta}\right)+\jmath S\left(\bar{u}_{\theta}\right)-\left(C\left(\underline{u}_{\theta}\right)+\jmath S\left(\underline{u}_{\theta}\right)\right) .
$$

Finally, the radiated power of a continuous line-source for a quadratic phase excitation can readily be expressed as follows,

$|S F(\theta)|^{2}=\frac{\pi I_{0}^{2}}{2 B_{2} L^{2}}\left[\left(C\left(\bar{u}_{\theta}\right)-C\left(\underline{u}_{\theta}\right)\right)^{2}+\left(S\left(\bar{u}_{\theta}\right)-S\left(\underline{u}_{\theta}\right)\right)^{2}\right]$.

This expression gives insight on the fact that the radiated power is governed by the behavior of Fresnel functions evaluated on $\underline{u}_{\theta}$ and $\bar{u}_{\theta}$, for $\theta \in[0, \pi]$. To intuitively understand how the quadratic phase variation is responsible for the beam widening phenomenon, it is interesting to express the difference $\Delta_{\bar{u}_{\theta}, \underline{u}_{\theta}}$ between the integral bounds, or equivalenlty the Fresnel function evaluation points, that is,

$$
\Delta_{\bar{u}_{\theta}, \underline{u}_{\theta}}=\bar{u}_{\theta}-\underline{u}_{\theta}=\sqrt{\frac{2 B_{2}}{\pi}} L .
$$

This integration interval remarkably depends on coefficient $B_{2}$, i.e the quadratic phase variation, for a given length source L. $\Delta_{\bar{u}_{\theta}, \underline{u}_{\theta}}$ is depicted in Figure 1 and 2, on which the left-hand plots correspond to the Fresnel functions, with a visualization of the interval range covered by variables $\underline{u}_{\theta}$ and $\bar{u}_{\theta}$ when $\theta$ goes from 0 to $\pi$. As observed when making the link with the right-hand figures, the beam shape results from the traveling of $\underline{u}_{\theta}$ and $\bar{u}_{\theta}$ points on the Fresnel functions. From Figure 1 and 2 , it is observed that $\Delta_{\bar{u}_{\theta}, \underline{u}_{\theta}}$, i.e the distance between $\bar{u}_{\theta}$ and $\underline{u}_{\theta}$, drives the angular distance between the couple of Fresnel integrals $\left(C\left(\bar{u}_{\theta}\right), S\left(\bar{u}_{\theta}\right)\right)$ and $\left(-C\left(\underline{u}_{\theta}\right),-S\left(\underline{u}_{\theta}\right)\right)$. Thus, the angular distance between the couple of Fresnel integrals can be tuned through $B_{2}$ for a given length source $L$. More precisely, the beam formation is triggered when $\underline{u}_{\theta}$ pass by the minima of the normalized cosine Fresnel function and released when $\bar{u}_{\theta}$ pass by the maxima of the normalized cosine Fresnel function. As can be observed comparing Figures 1 and 2 , the larger $\Delta_{\bar{u}_{\theta}, \underline{u}_{\theta}}$, the higher the angular distribution of the radiated power will be.

From this analysis, it turns out that $\Delta_{\bar{u}_{\theta}, \underline{u}_{\theta}}$ plays a central role in the adaptation of the beam width. However, its influence through the Fresnel functions and far-field radiation pattern is highly non-linear, which prevents a straightforward usage of Eq. (13) as a way to adapt parameter $B_{2}$ and tune the beam width. For example, although it is clear that the lowest $B_{2}$ value is zero, leading to a pure linear phase excitation (coming back to Eq. (6)), the upper limit is harder to define since $\underline{u}_{\theta}$ and $\bar{u}_{\theta}$ depend both on the length source $L$ and the steering angle $\theta_{\max }$. Hence, a deeper analysis of the influence of parameter $B_{2}$ has to be led to properly control the broadening effect.

\section{BEAM BROADENING CONTROL}

From the previous section, it is understood that the beam shape obtained from a quadratic excitation of a line-source depends on the evaluation intervals of the Fresnel integrals. Those intervals correspond to the ranges of $\underline{u}_{\theta}$ and $\bar{u}_{\theta}$ functions, both depending on the coefficients $B_{1}$ and $B_{2}$. Our goal is now to establish a formal expression making the link between such coefficients and the beam width. Due to the complexity of the manipulation of the transcendent Fresnel integrals, a complete closed form derivation is intractable to find such an expression. Some convenient approximation is however possible by considering the localization of the cosine Fresnel functions maxima.

\section{A. Fresnel angular distance}

As depicted in Figure 3, the studied beam width is highly correlated to the spacing between the maxima of the cosine Fresnel functions $C\left(\bar{u}_{\theta}\right)$ and $-C\left(\underline{u}_{\theta}\right)$. In contrast, the influence of the sine Fresnel functions seems to remain less significant. In the sequel, the spacing between the maxima of the cosine Fresnel functions $C\left(\bar{u}_{\theta}\right)$ and $-C\left(\underline{u}_{\theta}\right)$ is referred to as the Fresnel angular distance $\Delta_{F}$. The Fresnel angular distance is defined as,

$$
\Delta_{F}=\bar{\theta}_{\max }-\underline{\theta}_{\max },
$$

where,

$$
\left\{\begin{array}{l}
\bar{\theta}_{\max }=\operatorname{Arg} \max _{\theta} C\left(\bar{u}_{\theta}\right) \\
\underline{\theta}_{\max }=\operatorname{Arg} \max _{\theta}-C\left(\underline{u}_{\theta}\right)
\end{array} .\right.
$$




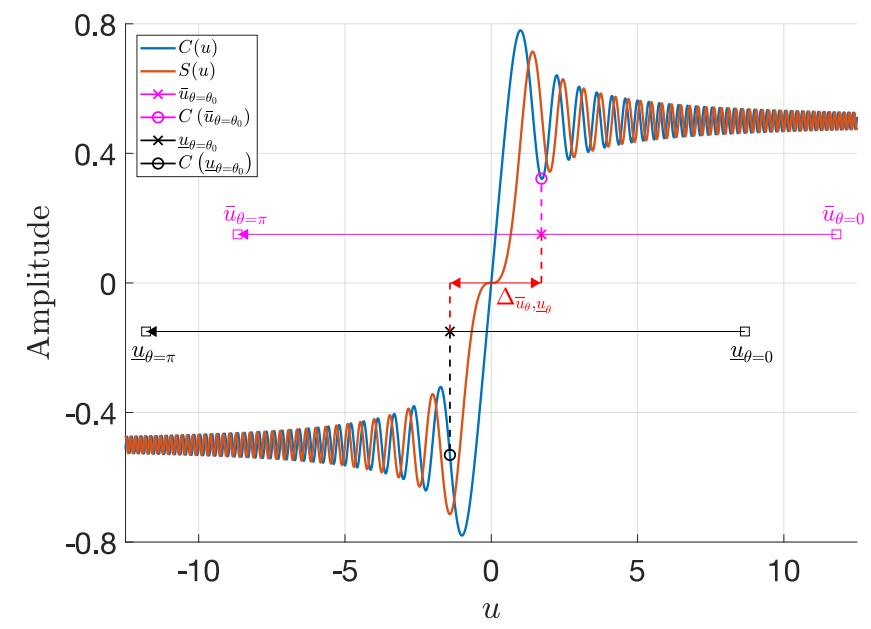

(a) Depiction of the normalized Fresnel functions $C(u)$ and $S(u)$ as well as the explored regions that correspond to $\bar{u}_{\theta}$ and $\underline{u}_{\theta}$ interval ranges

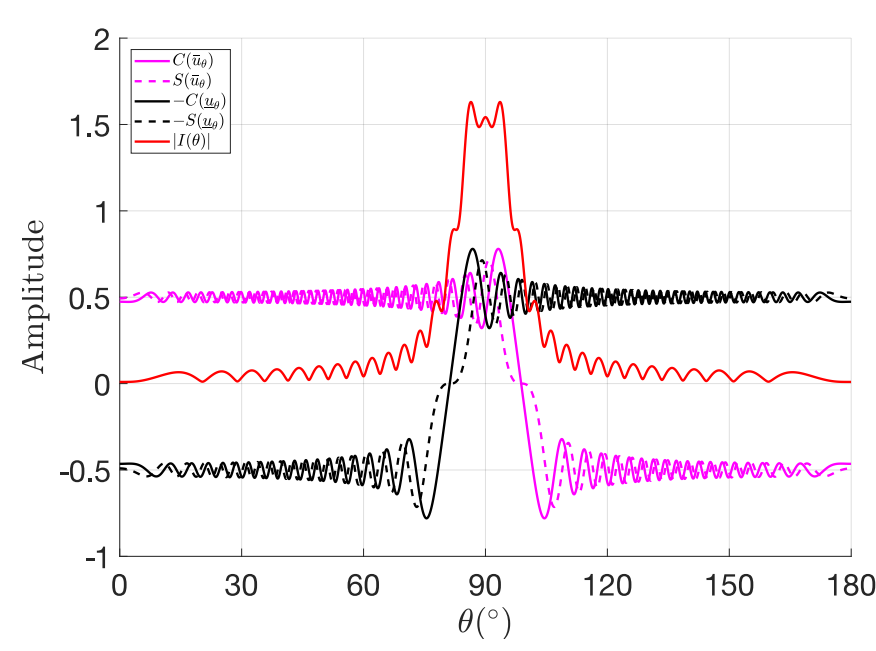

(b) Depiction of the Fresnel functions and of $|I(\theta)|$ according to the elevation angle $\theta$

Figure 1: Continuous line-source of length $L=16 \lambda(\lambda=0.01 \mathrm{~m})$ with input parameters $B_{1}=0$ and $B_{2}=600$

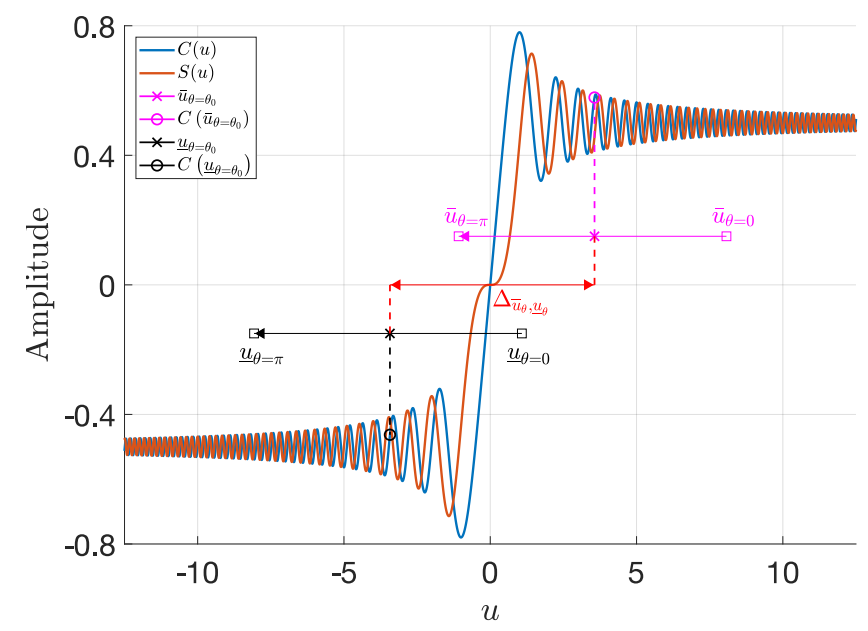

(a) Depiction of the normalized Fresnel functions $C(u)$ and $S(u)$ as well as the explored regions that correspond to $\bar{u}_{\theta}$ and $\underline{u}_{\theta}$ interval ranges

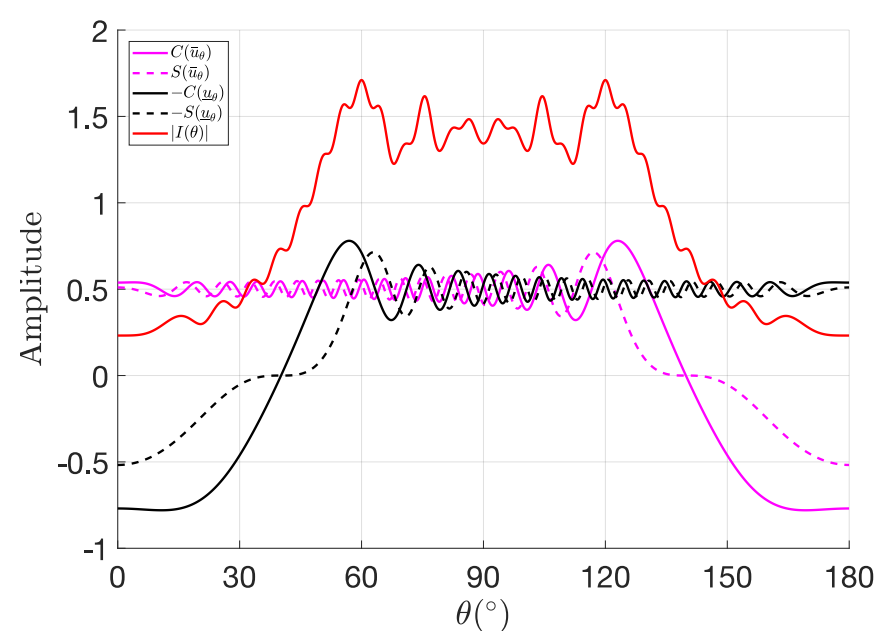

(b) Depiction of the Fresnel functions and of $|I(\theta)|$ according to the elevation angle $\theta$

Figure 2: Continuous line-source of length $L=16 \lambda(\lambda=0.01 \mathrm{~m})$ with input parameters $B_{1}=0$ and $B_{2}=3000$

$\Delta_{F}$ is expected to serve as a comprehensive dimensioning parameter, contrary to the function $\Delta_{\bar{u}_{\theta}, \underline{u}_{\theta}}$ previously introduced. As further illustrated in IV, one additional motivation for introducing $\Delta_{F}$ comes from the near linear relationship between that variable and the beam width of the phased array exploiting a quadratic phase excitation.

As cosine Fresnel functions $C(u)$ and $-C(u)$ take their global maxima at $u=1$ and $u=-1$, the analytical derivation of $\Delta_{F}$ can be obtained from the expression of variables $\underline{u}_{\theta}$ and $\bar{u}_{\theta}$ introduced in Eq. (10) by simply solving $\underline{u}_{\theta}=-1$ and $\bar{u}_{\theta}=1$. The Eq. (14) is then rewritten,

$$
\Delta_{F}=\arccos \left(x_{0}+\Delta_{x}\right)-\arccos \left(x_{0}-\Delta_{x}\right),
$$

with, $x_{0}=-B_{1} / k_{0}=\cos \left(\theta_{\max }\right)$ and,

$$
\Delta_{x}=\lambda \sqrt{\frac{B_{2}}{2 \pi}}\left(1-\frac{\Delta_{\bar{u}_{\theta}, \underline{u}_{\theta}}}{2}\right) .
$$

It appears that the Fresnel angular distance depends on both the targeted steering angle $\theta_{\max }$, through parameter $B_{1}$, and the length variation $\Delta_{x}$ as a function of the quadratic parameter $B_{2}$. One may notice that interval $\Delta_{\bar{u}_{\theta}, \underline{u}_{\theta}}$ is involved in the control of the Fresnel angular distance. However, the beam width adaptation through $\Delta_{\bar{u}_{\theta}, \underline{u}_{\theta}}$ is not straightforward since this function is related to $\Delta_{F}$ by a difference of nonlinear arccos functions. Hence, as already discussed in the previous section, adapting the beam width upon $\Delta_{\bar{u}_{\theta}, \underline{u}_{\theta}}$ only is not practical, whereas using $\Delta_{F}$ is of better convenience. 


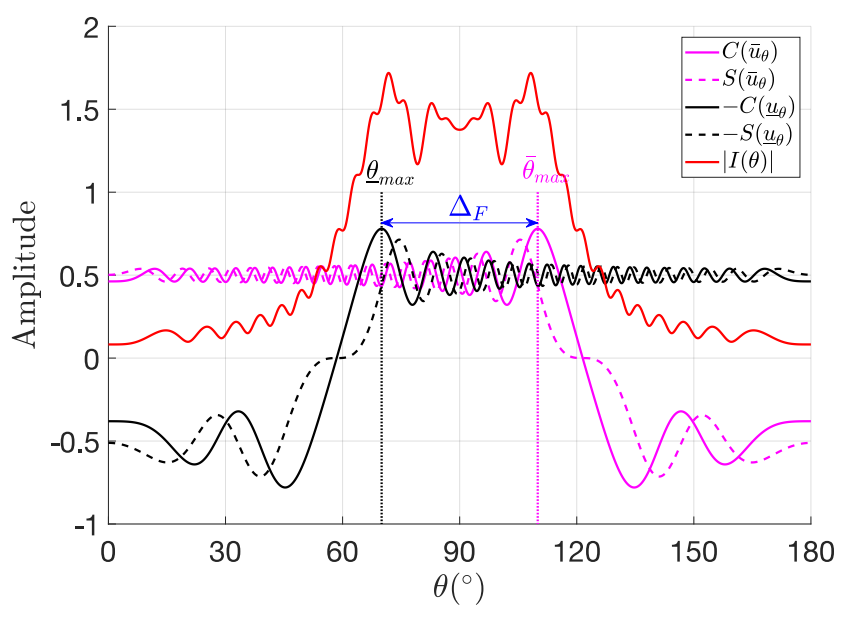

Figure 3: Highlight of the match between the maxima of the cosine Fresnel functions and the beam width of the far-field radiation pattern for a quadratic excitation of a line-source.

As additional comments, it is noticeable that the beam shape is symmetrical with respect to the boresight direction $\left(\theta_{\max }=90^{\circ}\right)$ for $B_{1}=0$ and $\Delta_{F}$ expression reduces to $\Delta_{F}=2 \arccos \left(\Delta_{x}\right)-\pi$. In other configurations however, i.e. for $B_{1} \neq 0$, the beam shape is no longer symmetrical about the steering angle $\theta_{\max }$. Remarkably, $\Delta_{F}=0$ for $B_{2}=\frac{2 \pi}{L^{2}}$. This configuration corresponds to the situation for which the maxima of the Fresnel functions coincides. In that case, the beam width is limited, but not strictly equivalent to the nominal beam width obtained with a pure linear phase excitation. This latter case is rather obtained when $B_{2}$ tends to 0 . In that case, $\Delta_{F}$ becomes negative, i.e. the maxima of the cosine Fresnel functions come in the opposite order than the one displayed in Figure 3. To summarize, we have the following definition range of $\Delta_{F}$,

$$
\left\{\begin{array}{l}
\Delta_{F}<0 \text { for } B_{2} \in\left[0, \frac{2 \pi}{L^{2}}[\right. \\
\Delta_{F} \geq 0 \text { for } B_{2} \geq \frac{2 \pi}{L^{2}}
\end{array},\right.
$$

where the first interval is obtained for $\Delta_{x}>0$ and the second for $\Delta_{x} \leq 0$.

On one other hand, it is important to keep in mind that the Eq. (16) assumes that the evaluated maxima in Eq. (15) correspond to the global maxima of the $C(u)$ and $-C(u)$ functions, which amounts to saying that the variables $\underline{u}_{\theta}$ and $\bar{u}_{\theta}$ span a range of values such that $\exists \theta \in[0, \pi], \underline{u}_{\theta}=-1$ and $\bar{u}_{\theta}=1$. These constraints are implicitly embedded in Eq. (16) by the definition range of the arccos functions that gives the range of values $\Delta_{F}$. Hence, for a given desired steering angle $\theta_{\max }, \Delta_{x}$ should be such that $-1 \leq x_{0}+\Delta_{x} \leq 1$ and $-1 \leq x_{0}-\Delta_{x} \leq 1$ are both verified. After further analyzing how these inequalities restrain the range of values for $\Delta_{F}$, it is determined that the maximum configurable value $\Delta_{F}^{\max }$ is expressed as,

$$
\left|\Delta_{F}\right| \leq \Delta_{F}^{\max }=\arccos (2 \cos \vartheta-1)
$$

with,

$$
\left\{\begin{array}{ll}
\vartheta=\theta_{\max } & \text { for } \theta_{\max } \in\left[0, \frac{\pi}{2}\right] \\
\vartheta=\pi-\theta_{\max } & \text { for } \theta_{\max } \in\left[\frac{\pi}{2}, \pi\right]
\end{array} .\right.
$$

Those two last equations may also be written as,

$$
\left|\Delta_{F}\right| \leq \Delta_{F}^{\max }\left(\theta_{\max }\right)=\arccos \left(2\left|x_{0}\right|-1\right) .
$$

This drives us to an important result that the Fresnel angular distance is limited to a maximum value directly depending on the desired steering angle $\theta_{\max }$. More specifically, we can conclude that the maximum Fresnel angular distance decreases as the steering angle increases.

\section{B. $B_{2}$ parameter adaptation}

The objective is now to express the coefficient $B_{2}$ according to the variable $\Delta_{F}$, in such a way that, in the end, $B_{2}$ can be computed from the desired Fresnel angular distance directly. To that purpose, one may rewrite (16) using the arccosine difference property defined as [23, p 80, Eq. (4.4.33)],

$$
\begin{aligned}
& \arccos (\alpha)-\arccos (\beta)=\operatorname{Sign}(\beta-\alpha) \times \\
& \quad \arccos \left(\alpha \beta+\sqrt{1-\alpha^{2}} \sqrt{1-\beta^{2}}\right), \forall \alpha, \beta \in[-1,1] .
\end{aligned}
$$

where $\operatorname{Sign}(x)$ denotes the sign function. This way, it is possible to express $\Delta_{x}$ as a function of $\Delta_{F}$. After simple manipulations we obtain,

$$
\Delta_{x}=-\operatorname{Sign}\left(\Delta_{F}\right) \sqrt{\frac{\left(1-\cos \Delta_{F}\right)\left(-2 x_{0}^{2}+1+\cos \Delta_{F}\right)}{2\left(1+\cos \Delta_{F}\right)}} .
$$

One may verify that the constraint on the maximum value of $\Delta_{F}$ given in Eq. (21) ensures that the argument of the square root is always positive, i.e. $\Delta_{x}$ always exists.

It is then straightforward to combine equations (13) and (17) to extract $B_{2}$ from a given $\Delta_{x}$. Indeed, $B_{2}$ merely consists in getting the roots of a quadratic form, which yields

$$
B_{2}=\frac{\pi}{\lambda L^{2}}\left(\lambda-2 L \Delta_{x}+\sqrt{\lambda\left(\lambda-4 L \Delta_{x}\right)}\right) .
$$

Through such formula, parameter $B_{2}$ is obtained from a computed $\Delta_{x}$ given by Eq. (22) for a desired $\Delta_{F}$, with again $\Delta_{F}$ satisfying (21). These last equations represent the mathematical statements upon which the proposed beam broadening design method presented in the next section is built.

Before getting further, it is however important to pay attention to the fact that additional constraints apply on $\Delta_{x}$ or equivalently on $\Delta_{F}$, to make Eq. (23) yield real values of $B_{2}$. Even if condition (21) is sufficient for $\Delta_{F} \geq 0$ since $\Delta_{x} \leq 0$ in that case, for $\Delta_{F}<0$ however, we have $\Delta_{x}>0$, and then we should impose,

$$
\Delta_{x} \leq \frac{\lambda}{4 L}
$$

After a complete derivation of such inequation involving Eq. (22), it is possible to identify the various conditions on $\Delta_{F}<0$ for Eq. (23) to hold whatever the selected steering angle $\theta_{\max }$. This is solved after classical though quite long 
mathematical derivations not detailed here. The results can be summarized as follows. Parameter $B_{2}$ computed for negative $\Delta_{F}$ is consistent if and only if,

$$
\Delta_{F}^{\min } \leq \Delta_{F}<0
$$

such that,

$\Delta_{F}^{\min }= \begin{cases}-\Delta_{F}^{\max }\left(\theta_{\max }\right) & \theta_{\max } \in\left[0, \theta_{0}\right] \cup\left[\pi-\theta_{0}, \pi\right] \\ -\arccos \left(c_{1} c_{2}+c_{3}\right) & \theta_{\max } \in\left[\theta_{0}, \pi-\theta_{0}\right]\end{cases}$

where $\theta_{0}=\arccos \left(1-\frac{\lambda}{4 L}\right)$, and,

$$
\left\{\begin{array}{l}
c_{1}=x_{0}-\frac{\lambda}{4 L} \\
c_{2}=x_{0}+\frac{\lambda}{4 L} \\
c_{3}=\sqrt{\left(c_{1}^{2}-1\right)\left(c_{2}^{2}-1\right)}
\end{array} .\right.
$$

Interestingly, for a source of large dimension compared to the wavelength, i.e. $L>>\lambda, \frac{\lambda}{4 L} \rightarrow 0$ and $\theta_{0} \rightarrow 0$. Consequently, we have $\Delta_{F}^{\min }=-\arccos \left(c_{1} c_{2}+c_{3}\right), \forall \theta_{\max } \in$ $[0, \pi]$. Meanwhile, if $L>>\lambda, c_{2} \rightarrow c_{1}$, and hence one can easily find that $c_{1} c_{2}+c_{3} \rightarrow 1$. This finally leads to $\Delta_{F}^{\min } \rightarrow 0$. This analysis allows to anticipate and give insight to a later observation showing that for large antenna arrays, the beam broadening effect is essentially achievable for positive $\Delta_{F}$.

\section{Practical Beam Broadening Method for ANTENNA ARRAYS}

From the previous developments, we have demonstrated that the $B_{2}$ coefficient of a quadratic phase excitation applied to a line source can be calculated according to the newly defined $\Delta_{F}$ variable whatever the source length $L$ and the steering angle $\theta_{\max }$. We now intend to exploit such results to propose a simple way for controlling the beam width of discrete linear antenna arrays. Remember that $\Delta_{F}$ is not proven to be strictly equal to the beam width, but has rather been proposed as a practical and representative parameter of it. In this section then, our goal is to study in which extend some calibration or correction process may be elaborated to make $\Delta_{F}$ be an actual tuning parameter of the beam width, and propose hereby a systematic method for beam broadening. In particular, as shown in the sequel, $\Delta_{F}$ turns out to be almost linearly depending on the so-called beam efficiency used as a reference metrics to characterize the broadening of the steered beams.

\section{A. Discrete-element array excitation}

As a preliminary step, let us remind that the mathematical proofs derived in Section III about the Fresnel angular distance control through parameter $B_{2}$ consider the radiation characteristics of a continuous source as established in Section II. In practical scenarios however, discrete-element arrays controlled by an integer number of phase shifters are rather used. Nevertheless, the radiation characteristics of a dicreteelement array can be approximated by those of a continuous source, making the beam broadening phenomenom defined in the previous sections still valid in spite of the discretization [12, Chapter 7.2]. In the sequel, we consider a linear antenna

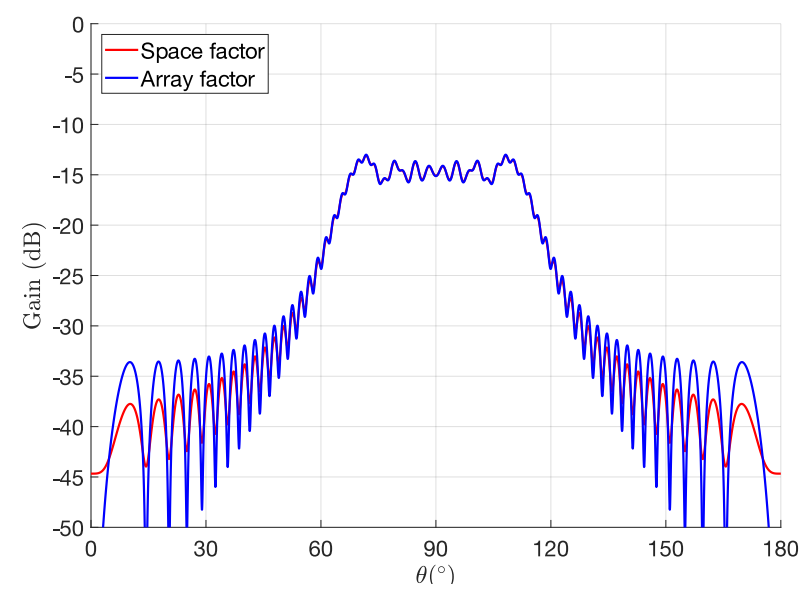

Figure 4: Far-field radiation pattern produced by a continuous line-source $(L=32 \lambda)$ and by a discrete-element array $(M=$ 64 ) for the same quadratic phase excitation law

array, placed along the z-axis, that is composed of $M$ antenna elements and controlled by a quadratic phase excitation. The array factor $A F(\theta)$, that is the twin of the space factor $S F(\theta)$ for discrete arrays, is then given by

$$
A F(\theta)=\frac{1}{\sqrt{M}} \sum_{m=1}^{M} e^{j\left(k_{0} z_{m} \cos (\theta)+\phi_{m}\right)}
$$

with

$$
\phi_{m}=B_{2}\left(z_{m}-\frac{z_{1}+z_{M}}{2}\right)^{2}+B_{1} z_{m}
$$

being the quadratic phase excitation. The position of the $n$th antenna element is given by $z_{m}=(m-1) d, d$ being the interelement spacing. Without loss of generality, we will consider $d=\frac{\lambda}{2}$ in the sequel.

As depicted in Figure 4, the line-source discretization mainly induces higher sidelobes but the beamwidth is kept the same. The coefficient parameterization derived for linesources is thus kept unchanged in the case of linear antenna arrays.

\section{B. Beam width characterization}

To go further, we need an appropriate metrics to measure the width of the beam produced by the antenna array. As mentioned in the introduction, ripples can exceed $3 \mathrm{~dB}$ when using a pure phase excitation. Thus, relying on the conventional $3 \mathrm{~dB}$ beam width definition is not convenient in our case. We then propose to characterize the beam width from the so-called beam efficiency of the array [12, Chapter 2.10]. The beam efficiency of an antenna may be defined as,

$$
\eta_{B}=\frac{\int_{\theta_{b}-\frac{\Omega_{b}}{2}}^{\theta_{b}+\frac{\Omega_{b}}{2}}|A F(\theta)|^{2} \sin (\theta) d \theta}{\int_{0}^{\pi}|A F(\theta)|^{2} \sin (\theta) d \theta} .
$$

It represents the ratio of the power radiated within a solid angle $\Omega_{b}$ around a main direction $\theta_{b}$ to the total power radiated. Strictly speaking, the radiated power should be 


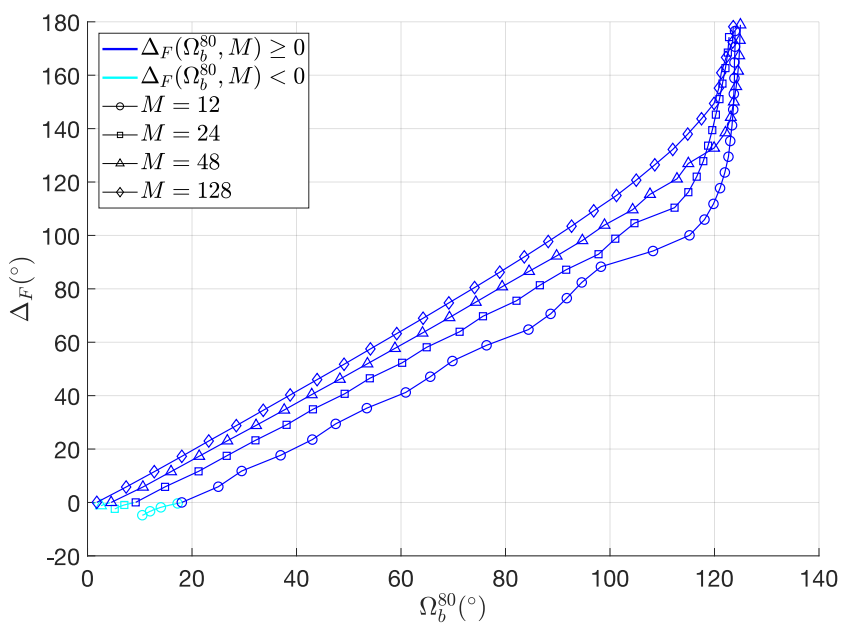

(a) $\Delta_{F} \in\left[-20^{\circ}, 180^{\circ}\right]$

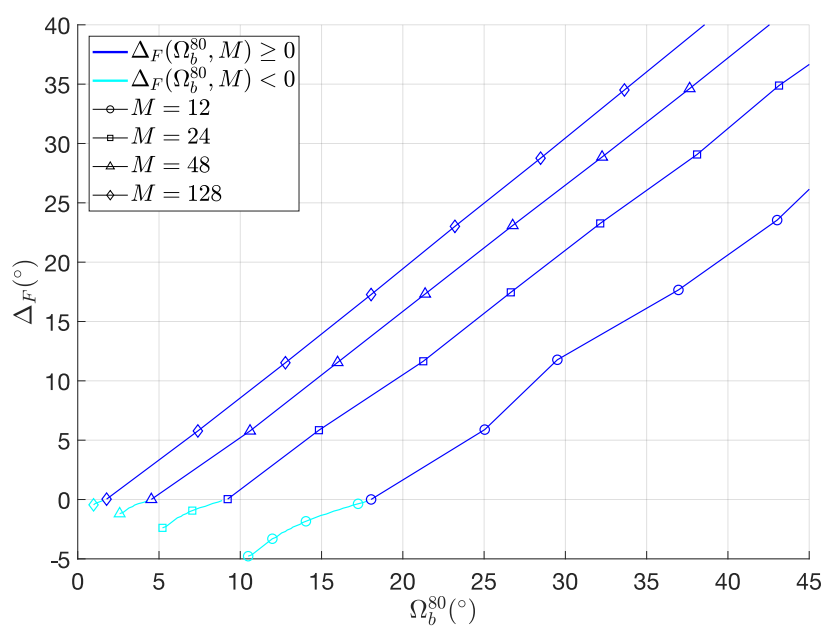

(b) $\Delta_{F} \in\left[-5^{\circ}, 40^{\circ}\right]$

Figure 5: $\Delta_{F}$ versus $\Omega_{b}^{80}$ for $M \in\{12,24,48,128\}$

measured around the barycenter of the power pattern, i.e at the angular value that splits the power pattern in such a way that $50 \%$ percent of the power is radiated on each angular sector. Hence, the actual steering direction $\theta_{b}$ to consider is such that,

$$
\int_{0}^{\theta_{b}}|A F(\theta)|^{2} \sin (\theta) d \theta=\int_{\theta_{b}}^{\pi}|A F(\theta)|^{2} \sin (\theta) d \theta .
$$

The barycenter is an essential metrics for ensuring that the radiated power is focused in the right direction, which may be slightly different from the initial steering angle $\theta_{\max }$ as discussed later on.

From these considerations, it is then possible to define the beam width $\Omega_{b}^{X}$ of the array for a target beam efficiency of $X \%$, that is,

$$
\Omega_{b}^{X}=\arg _{\Omega_{b}} \eta_{B}=X \% .
$$

It simply represents the angular range around $\theta_{b}$ within which $X \%$ of the total power is radiated. Hence, the beam width has not a unique value, but rather depends on the targeted beam efficiency. In the following sections, we consider that $X=80$ but the described method could be repeated for other $X$ values.

In the next sections, further details are provided regarding $\theta_{b}$ and the relationship between $\Delta_{F}$ and $\Omega_{b}^{X}$ for boresight and non-boresight directions.

\section{Beam width control for boresight direction}

For the boresight direction, i.e $\theta_{\max }=\pi / 2$, the first thing to notice is that $\theta_{b}=\pi / 2$ regardless of the $\Delta_{F}$ value. Indeed, since $\theta_{\max }=\pi / 2, B_{1}$ is null and $\phi_{n}$ is an even function resulting in a symmetrical power pattern whose barycenter coincides with the steering angle $\theta_{\max }$.

As already discussed, $\Delta_{F}$ is a practical parameter strongly linked to the width of the beam but is not formally expressed as a function of it. Therefore, we propose to investigate about the possible relationship between $\Delta_{F}$ and the previously defined beam width $\Omega_{b}^{X}$ for an antenna array composed of $M$ antennas. The following procedure was used :
1- Set the variation range of $\Delta_{F}$ for $M$ antennas from Eq. (21) and Eq. (25) as,

$$
\Delta_{F} \in\left[-\arccos \left(1-\frac{1}{2 M^{2}}\right), \pi[\right.
$$

2- Compute $B_{2}$ coefficient using Eq. (23) for each $\Delta_{F}$.

3 - Compute the phase law $\phi_{m}$ from Eq. (28) for each $\Delta_{F}$.

4- Compute the array factor $A F(\theta)$ for each phase law using Eq. (27).

$5-$ Find $\Omega_{b}^{X}$ by solving Eq. (29) numerically for each $A F(\theta)$ obtained from each $\Delta_{F}$.

In Figure 5, we plot $\Delta_{F}\left(\Omega_{b}^{80}, M\right)$ as a function of the obtained beam width $\Omega_{b}^{80}$ for various array sizes $M$. An almost linear relationship is observed between the two parameters, at least until a maximum value $\Omega_{\max }^{80}(M)$ of the beam width above which $\Delta_{F}\left(\Omega_{b}^{80}, M\right)$ rapidly goes to $\pi \operatorname{rad}$. Interestingly, the larger the antenna array, the more linear the relationship. More precisely, we can state that,

$$
\lim _{M \rightarrow \infty} \Delta_{F}\left(\Omega_{b}^{100}, M\right)=\Omega_{b}^{100}
$$

meaning that $\Delta_{F}$ asymptotically matches the beam width. Indeed the $I(\theta)$ function tends to be rectangular as $M$ grows due to the compression of the Fresnel functions $C\left(\bar{u}_{\theta}\right)$ and $-C\left(\underline{u}_{\theta}\right)$ [24]. Consequently, for large $M$ values, $\underline{\theta}_{\max }$ and $\bar{\theta}_{\max }$ perfectly coincide with the edge of a rectangle which width directly corresponds to the beam width $\Omega_{b}^{100}$. Moreover, $\Delta_{F}^{\min }$ tends to zero (recall discussion after Eq. (26)) as it is depicted in Figure $5 \mathrm{~b}$ where the range of values for $\Delta_{F}\left(\Omega_{b}^{80}, M\right)$ progressively becomes strictly positive when $M$ grows.

As we may apply our approach for millimeter transmission scenario, we are interested in the situation where $M$ is not too high. It is hence important to study the beam width control for $\Delta_{F}\left(\Omega_{b}^{X}, M\right) \geq 0$ as well as $\Delta_{F}\left(\Omega_{b}^{X}, M\right)<0$. As the relationship is asymptotically linear, we suggest to approximate it by a linear polynomial even at small $M$. As 


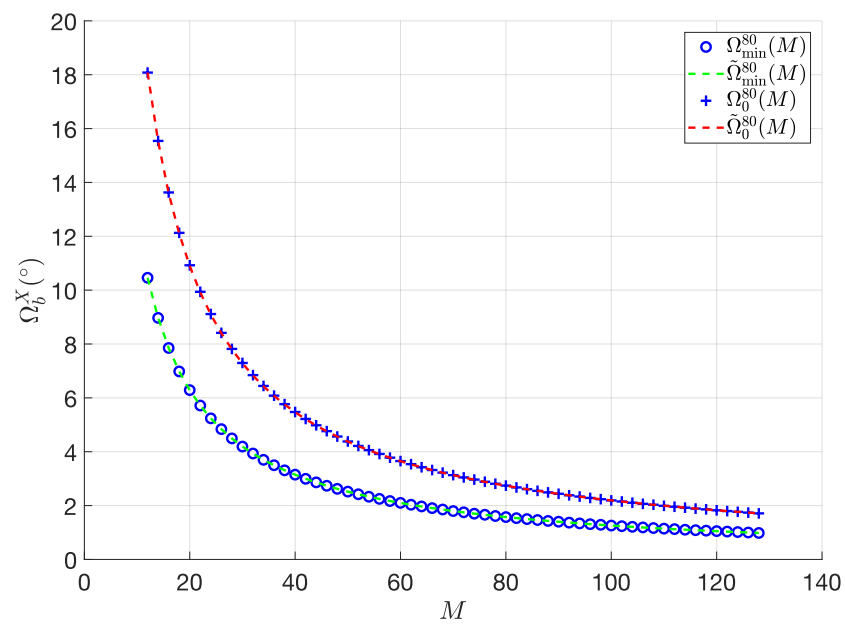

Figure 6: $\Omega_{\min / 0}^{80}(M)$ and $\tilde{\Omega}_{\min / 0}^{80}(M)$ for $M \in[12,128]$

may be noticed from Figure 5b, the average slope of the function is slightly different for positive and negative values of $\Delta_{F}\left(\Omega_{b}^{80}, M\right)$. Hence, each linear approximation may be studied individually as follows.

1) Linear approximation for $\Delta_{F}\left(\Omega_{b}^{X}, M\right)<0$ : In this case we have $\Delta_{F} \in\left[\Delta_{F}^{\min }(M), 0\left[\right.\right.$, with $\Delta_{F}^{\min }(M)=$ $-\arccos \left(1-\frac{1}{2 M^{2}}\right)$. Accordingly, let $\Omega_{0}^{X}(M)$ and $\Omega_{\min }^{X}(M)$ denote the beam width values respectively associated with $\Delta_{F}=0$ and $\Delta_{F}=\Delta_{F}^{\min }(M)$. The linear relationship then writes,

$$
\begin{aligned}
& \tilde{\Delta}_{F}\left(\Omega_{b}^{X}, M\right) \approx \Delta_{F}\left(\Omega_{b}^{X}, M\right) \\
& =\Delta_{F}^{\min }(M) \times\left(\frac{\Omega_{0}^{X}(M)-\Omega_{b}^{X}}{\Omega_{0}^{X}(M)-\Omega_{\min }^{X}(M)}\right), \\
& \Omega_{b}^{X} \in\left[\Omega_{\min }^{X}(M), \Omega_{0}^{X}(M)[.\right.
\end{aligned}
$$

It follows that if $\Omega_{0}^{X}(M)$ and $\Omega_{\text {min }}^{X}(M)$ are known $\forall M$, then Eq. (33) directly gives the adequate $\Delta_{F}$ for a targeted beam width $\Omega_{b}^{X}$ and a given array size $M$. A numerical study of the variation of $\Omega_{0}^{80}$ and $\Omega_{\mathrm{min}}^{80}$ versus $M$ yields the curves depicted in Figure 6. It is observed that both functions may be approached by the multiplicative inverse of a linear polynomial, that is,

$$
\tilde{\Omega}^{X}(M)=\frac{1}{\alpha M+\beta} \quad(\mathrm{rad}),
$$

with $\alpha$ and $\beta$ some real scalar values, depending on $X$. Through a least square fitting to such model for $X=80 \%$ for instance, we get the following approximations,

$$
\left\{\begin{array}{ll}
\tilde{\Omega}_{\min }^{80}(M)=\frac{1}{0.4548 \times M+2.05 \times 10^{-2}} & (\mathrm{rad}) \\
\tilde{\Omega}_{0}^{80}(M)=\frac{1}{0.2610 \times M+3.67 \times 10^{-2}} & (\mathrm{rad})
\end{array}, M \in[12,128]\right.
$$

The tightness of the proposed model is validated in Figure 6 where we observe that the beam width values $\tilde{\Omega}_{\min / 0}^{80}(M)$ generated by Eq. (35) are perfectly matching the actual ones

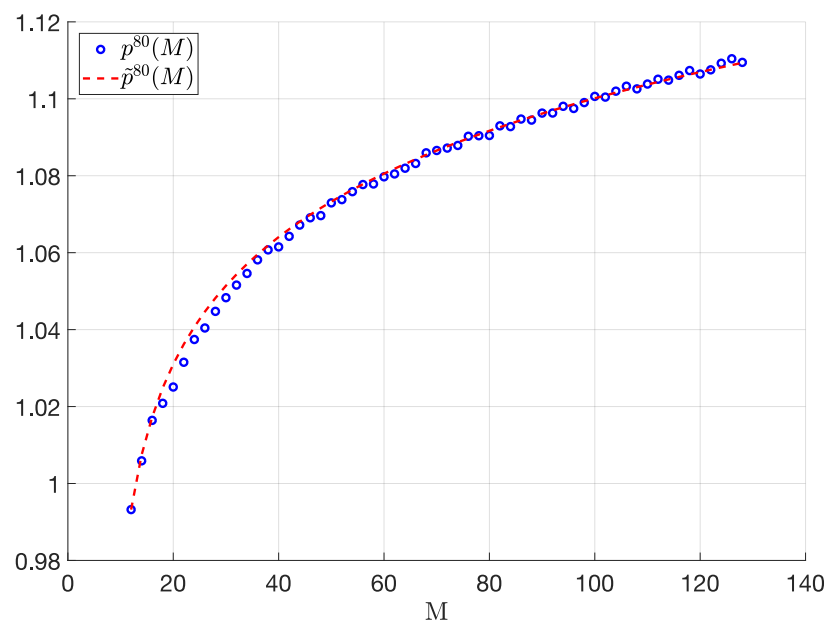

Figure 7: $p^{80}(M)$ and $\tilde{p}^{80}(M)$ for $M \in[12,128]$

obtained by simulations. We then conclude that $\Delta_{F}\left(\Omega_{b}^{80}, M\right)$ can accurately be approximated using Eq. (33) by substituting $\Omega_{0}^{X}(M)$ and $\Omega_{\min }^{X}(M)$ by the approached values given by Eq. (35). This methodology may be repeated for an other $\Omega_{b}^{X}$, e.g. $X=75 \%$ or $X=90 \%$.

2) Linear approximation for $\Delta_{F}\left(\Omega_{b}^{X}, M\right) \geq 0$ : For $\Delta_{F}\left(\Omega_{b}^{X}, M\right) \geq 0$, we assume that $\Omega_{F}^{X} \in\left[\Omega_{0}^{X}(M), \Omega_{\max }^{X}\right]$ with $\Omega_{0}^{X}(M)$ already given by Eq. (35) and $\Omega_{\max }^{X}$ chosen equal to $90^{\circ}$. As observed in Figure 5, the latter corresponds to a reasonable maximum value that enables the configuration of a large beam width while still ensuring a near linear relationship between $\Delta_{F}\left(\Omega_{b}^{80}, M\right)$ and $\Omega_{b}^{80}$ whatever $M$. We may choose a higher value of $\Omega_{\max }^{X}$, possibly reaching a sector of $120^{\circ}$, for arrays of several hundreds of antenna elements. On this basis, we may approximate $\Delta_{F}\left(\Omega_{b}^{X}, M\right)$ as a linear function as,

$$
\begin{aligned}
& \tilde{\Delta}_{F}\left(\Omega_{b}^{X}, M\right) \approx \Delta_{F}\left(\Omega_{b}^{X}, M\right) \\
& =p^{X}(M) \times\left(\Omega_{b}^{X}-\Omega_{0}^{X}(M)\right), \Omega_{b}^{X} \in\left[\Omega_{0}^{X}(M), \Omega_{\max }^{X}\right],
\end{aligned}
$$

where $p^{X}(M)$ is the slope of the linear polynomial for a given array size $M \cdot p^{X}(M)$ is determined numerically by means of linear regression analysis and is depicted in Figure 7 for $M \in[12,128]$. This function may be approximated by $\tilde{p}^{80}(M)$ which is expressed as,

$$
\tilde{p}^{80}(M)=\sqrt[32]{0.2317 \times M-1.9761}, M \in[12,128] .
$$

To summarize, the beam width $\Omega_{b}^{X}$ ranges from $\Omega_{\min }^{X}(M)$ to $\Omega_{\max }^{X}=90^{\circ}$ and is related to $\Delta_{F}\left(\Omega_{b}^{X}, M\right)$ through the function $\tilde{\Delta}_{F}\left(\Omega_{b}^{X}, M\right)$ by means of two linear approximations given by the Eq. 33 and 36. Interestingly, the terms composing the Eq. 33 and 36 , i.e $\Omega_{\min }^{X}(M), \Omega_{0}^{X}(M)$ and $p^{X}(M)$ can be accurately approximated by the Eq. 35 and 37 for $M \in[12,128]$ and $X=80 \%$. Therefore, a systematic relation connect the beam width $\Omega_{b}^{80}$ to the Fresnel angular distance $\Delta_{F}$. As a final step, the quadratic phase law can be computed 


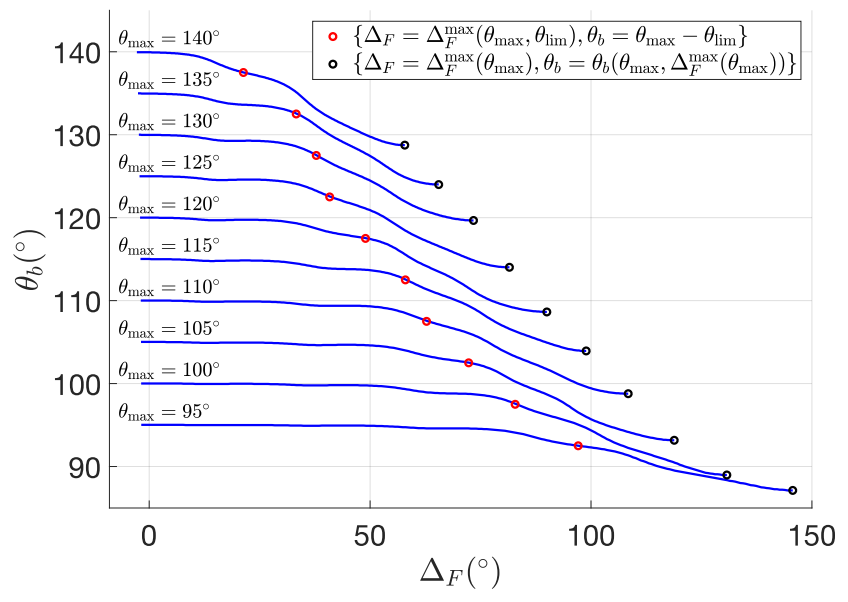

Figure 8: $\theta_{b}\left(\theta_{\max }, \Delta_{F}\right)$ according to $\Delta_{F}$ for various steering angles $\theta_{\max }$ considering an antenna array composed of $M=$ 32 antenna elements and a maximum drift $\theta_{\lim }=2.5^{\circ}$

Table I: Coefficient values for $M=32$ and $\theta_{\lim }=2.5^{\circ}$

\begin{tabular}{|c|c|c|}
\hline$\theta_{\max }$ & $\Delta_{F}^{\max }\left(\theta_{\max }, \theta_{\lim }\right)(\mathrm{rad})$ & $\Omega_{\max }^{80}\left(\theta_{\max }, \theta_{\lim }\right)(\mathrm{rad})$ \\
\hline $85^{\circ} / 95^{\circ}$ & 1.6947 & 1.7035 \\
\hline $80^{\circ} / 100^{\circ}$ & 1.4457 & 1.4905 \\
\hline $75^{\circ} / 105^{\circ}$ & 1.2620 & 1.3285 \\
\hline $70^{\circ} / 110^{\circ}$ & 1.0958 & 1.1885 \\
\hline $65^{\circ} / 115^{\circ}$ & 1.0120 & 1.1185 \\
\hline $60^{\circ} / 120^{\circ}$ & 0.8545 & 0.9885 \\
\hline $55^{\circ} / 125^{\circ}$ & 0.7130 & 0.8705 \\
\hline $50^{\circ} / 130^{\circ}$ & 0.6600 & 0.8445 \\
\hline $45^{\circ} / 135^{\circ}$ & 0.5808 & 0.7845 \\
\hline $40^{\circ} / 140^{\circ}$ & 0.3722 & 0.6025 \\
\hline
\end{tabular}

systematically according to the desired $\Omega_{b}^{80}$ since the quadratic coefficient $B_{2}$ is related to $\Delta_{F}$, recalling the Eq.22 and 23 .

\section{Non-boresight directions}

For non-boresight directions, i.e $\theta_{\max } \neq \pi / 2, \phi_{m}$ is no longer an even function since $B_{1}$ introduces a linear phase shift. The resulting power pattern is asymmetric and the barycenter $\theta_{b}$ depends on the $\Delta_{F}$. As previously discussed, the barycenter is an essential aspect so it is important to ensure that the drift between the desired steering angle $\theta_{\max }$ and the effective one, i.e the barycenter $\theta_{b}$, is not too high.

For simplification purposes, we consider an antenna array comprising $M=32$ antenna elements. In addition, we assume that $\theta_{\max }$ ranges from $40^{\circ}$ to $140^{\circ}$ with an angular resolution of $5^{\circ}$. Considering those parameters, the barycenter $\theta_{b}\left(\theta_{\max }, \Delta_{F}\right)$ is evaluated numerically for $\Delta_{F} \in$ $\left[\Delta_{F}^{\min }\left(\theta_{\max }\right), \Delta_{F}^{\max }\left(\theta_{\max }\right)\right]$, recalling the Eq.21 and 25. As depicted in Figure 8, the drift between $\theta_{\max }$ and $\theta_{b}\left(\theta_{\max }, \Delta_{F}\right)$ increases with $\Delta_{F}$. Consequently, a trade-off exists between the maximum desired drift $\theta_{\mathrm{lim}}$ and the maximum configurable beam width $\Omega_{\max }^{X}\left(\theta_{\max }\right)$. In order to ensure that the maximum effective drift doesn't exceed $\theta_{\text {lim }}$, we determine numerically the maximum Fresnel angular distance $\Delta_{F}^{\max }\left(\theta_{\max }, \theta_{\text {lim }}\right)$ which ensures that,

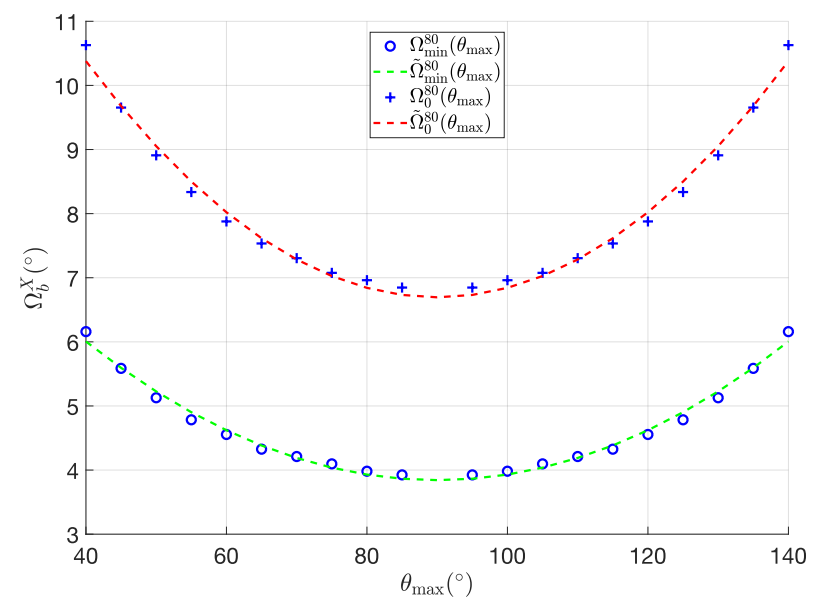

Figure 9: $\Omega_{\min / 0}^{80}\left(\theta_{\max }\right)$ and $\tilde{\Omega}_{\min / 0}^{80}\left(\theta_{\max }\right)$ for $\theta_{\max } \in$ $[40,140]$ and $M=32$

$$
\begin{array}{r}
\left|\theta_{b}\left(\theta_{\max }, \Delta_{F}\right)-\theta_{\max }\right| \leq \theta_{\lim }, \\
\Delta_{F} \in\left[\Delta_{F}^{\min }\left(\theta_{\max }\right), \Delta_{F}^{\max }\left(\theta_{\max }, \theta_{\lim }\right)\right] .
\end{array}
$$

In the suggested implementation, a maximum drift $\theta_{\lim }$ of $2.5^{\circ}$ is chosen owing to the $5^{\circ}$ angular resolution of $\theta_{\max }$. The values taken by $\Delta_{F}^{\max }\left(\theta_{\max }, \theta_{\lim }=2.5^{\circ}\right)$ for $M=32$ are illustrated in Figure 8 and given in Table I.

In order to determine the relationship between $\Delta_{F}\left(\Omega_{b}^{X}, \theta_{\max }, \theta_{\text {lim }}\right)$ and $\Omega_{b}^{X}$ for non-boresight directions, the 5 -step procedure presented in section (IV-C) is applied, with respect to $\Delta_{F} \in\left[\Delta_{F}^{\min }\left(\theta_{\max }\right), \Delta_{F}^{\max }\left(\theta_{\max }, \theta_{\mathrm{lim}}=2.5^{\circ}\right)\right]$. In a similar manner, the relation between $\Delta_{F}\left(\Omega_{b}^{X}, \theta_{\max }, \theta_{\text {lim }}\right)$ and $\Omega_{b}^{X}$ is approximated by means of two linear approximations.

1) Linear approximation for $\Delta_{F}\left(\Omega_{b}^{X}, \theta_{\max }, \theta_{\lim }\right)<0$ :

Following the same approach as the one described in Section IV-C for $\Delta_{F}\left(\Omega_{b}^{X}, M\right)<0$ estimate, $\Delta_{F}\left(\Omega_{b}^{X}, \theta_{\max }, \theta_{\text {lim }}\right)$ may be approximated by,

$$
\begin{aligned}
& \tilde{\Delta}_{F}\left(\Omega_{b}^{X}, \theta_{\max }\right) \approx \Delta_{F}\left(\Omega_{b}^{X}, \theta_{\max }, \theta_{\lim }\right) \\
& =\Delta_{F}^{\min }\left(\theta_{\max }\right) \times\left(\frac{\Omega_{0}^{X}\left(\theta_{\max }\right)-\Omega_{b}^{X}}{\Omega_{0}^{X}\left(\theta_{\max }\right)-\Omega_{\min }^{X}\left(\theta_{\max }\right)}\right), \\
& \Omega_{b}^{X} \in\left[\Omega_{\min }^{X}\left(\theta_{\max }\right), \Omega_{0}^{X}\left(\theta_{\max }\right)[.\right.
\end{aligned}
$$

A numerical study of the variation of $\Omega_{0}^{80}$ and $\Omega_{\text {min }}^{80}$ versus $\theta_{\max }$ yields the curves depicted in Figure 9. It is observed that both functions may be approached by quadratic functions, that is,

$$
\tilde{\Omega}^{X}\left(\theta_{\max }\right)=\alpha \times \theta_{\max }^{2}+\beta \times \theta_{\max }+\gamma \quad(\mathrm{rad}),
$$

with $\alpha, \beta$ and $\gamma$ some real scalar values, depending on $X$. Through a least square fitting to such model for $X=80 \%$ for instance, we get the following approximations, 


$$
\left\{\begin{array}{l}
\tilde{\Omega}_{\min }^{80}\left(\theta_{\max }\right)=0.0496 \times \theta_{\max }^{2}-0.1557 \times \theta_{\max }+0.1893 \\
\tilde{\Omega}_{0}^{80}\left(\theta_{\max }\right)=0.0844 \times \theta_{\max }^{2}-0.2653 \times \theta_{\max }+0.3252 \\
\theta_{\max } \in\left[40^{\circ}, 140^{\circ}\right]
\end{array},\right.
$$

The tightness of the proposed model is validated in Figure 9 where we observe that the beam width values $\tilde{\Omega}_{\min / 0}^{80}\left(\theta_{\max }\right)$ generated by Eq. (41) match the ones obtained by simulations with fair accuracy. We then conclude that $\Delta_{F}\left(\Omega_{b}^{80}, \theta_{\max }, \theta_{\lim }\right)$ can be approximated using Eq. (39) by substituting $\Omega_{0}^{X}\left(\theta_{\max }\right)$ and $\Omega_{\min }^{X}\left(\theta_{\max }\right)$ by the approached values given by Eq. (41). This methodology may be repeated for an other $\Omega_{b}^{X}$, e.g. $X=$ $75 \%$ or $X=90 \%$.

2) Linear approximation for $\Delta_{F}\left(\Omega_{b}^{X}, \theta_{\max }, \theta_{\lim }\right) \geq 0$ : The general idea described in Section IV-C for $\Delta_{F}\left(\Omega_{b}^{X}, M\right) \geq$ 0 is followed. The main difference is that the maximum beam width $\Omega_{\max }^{X}\left(\theta_{\max }, \theta_{\mathrm{lim}}\right)$ depends on the steering angle $\theta_{\max }$ and on the maximum desired drift $\theta_{\lim }$, while $\Omega_{\max }^{X}$ is constant ( $M$-invariant) in Section IV-C. Hence, $\Delta_{F}\left(\Omega_{b}^{X}, \theta_{\max }, \theta_{\lim }\right)$ may be approximated by,

$$
\begin{aligned}
& \tilde{\Delta}_{F}\left(\Omega_{b}^{X}, \theta_{\max }, \theta_{\lim }\right) \approx \Delta_{F}\left(\Omega_{b}^{X}, \theta_{\max }, \theta_{\lim }\right) \\
& =p^{X}\left(\theta_{\max }, \theta_{\lim }\right) \times\left(\Omega_{b}^{X}-\Omega_{0}^{X}\left(\theta_{\max }\right)\right), \\
& \Omega_{b}^{X} \in\left[\Omega_{0}^{X}\left(\theta_{\max }\right), \Omega_{\max }^{X}\left(\theta_{\max }, \theta_{\lim }\right)\right],
\end{aligned}
$$

where $p^{X}\left(\theta_{\max }, \theta_{\lim }\right)$ is the slope of the linear polynomial for a given steering angle $\theta_{\max }$ and a desired maximum drift $\theta_{\text {lim }}$. In contrast with the method described in Section IV-C, $p^{X}\left(\theta_{\max }, \theta_{\mathrm{lim}}\right)$ is not approximated by means of a linear regression analysis. Indeed, the following estimate,

$$
p^{X}\left(\theta_{\max }, \theta_{\lim }\right)=\frac{\Delta_{F}^{\max }\left(\theta_{\max }, \theta_{\mathrm{lim}}\right)}{\Omega_{\max }^{X}\left(\theta_{\max }, \theta_{\lim }\right)-\Omega_{0}^{X}\left(\theta_{\max }\right)}
$$

is more appropriate to ensure that $\tilde{\Delta}_{F}\left(\Omega_{b}^{X}, \theta_{\max }, \theta_{\text {lim }}\right)$ doesn't exceed $\Delta_{F}^{\max }\left(\theta_{\max }, \theta_{\text {lim }}\right)$ and thus respect the expected maximum drift $\theta_{\mathrm{lim}}$. Note that $\Omega_{\max }^{80}\left(\theta_{\max }, \theta_{\mathrm{lim}}=2.5^{\circ}\right)$ values have been estimated numerically for $M=32$ and are given in Table I.

To summarize, the beam width $\Omega_{b}^{X}$ ranges from $\Omega_{\min }^{X}\left(\theta_{\max }\right)$ to $\Omega_{\max }^{X}\left(\theta_{\max }, \theta_{\lim }\right)$ and is related to $\Delta_{F}\left(\Omega_{b}^{X}, \theta_{\max }, \theta_{\lim }\right)$ through the function $\tilde{\Delta}_{F}\left(\Omega_{b}^{X}, \theta_{\max }, \theta_{\lim }\right)$ by means of two linear approximations given by the Eq. 39 and 42. The maximum Fresnel angular distance $\Delta_{F}^{\max }\left(\theta_{\max }, \theta_{\text {lim }}\right)$ in the Eq. 43 limits the maximum drift to $\theta_{\lim }$ and has to be determined numerically in conjunction with $\Omega_{\max }^{X}\left(\theta_{\max }, \theta_{\text {lim }}\right)$ for the steering angles $\theta_{\max }$ of interest. Remarkably, the terms $\Omega_{\min }^{X}\left(\theta_{\max }\right)$ and $\Omega_{0}^{X}\left(\theta_{\max }\right)$ can be approximated with fair accuracy by the Eq. 41 for $\theta_{\max } \in\left[40^{\circ}, 140^{\circ}\right]$ and $X=80 \%$, considering an antenna array composed of $M=32$ antenna elements. Therefore, a systematic relation connect the beam width $\Omega_{b}^{80}$ to the Fresnel angular distance $\Delta_{F}$ for a given steering angle $\theta_{\max }$ and desired maximum drift $\theta_{\lim }$. As a final step, the quadratic phase law can be computed systematically according to the desired $\Omega_{b}^{80}$ since the quadratic coefficient $B_{2}$ is related to $\Delta_{F}$ by means of the Eq.22 and 23.

\section{NUMERICAL RESULTS}

In the last section, a 5-step procedure that enables to determine the relation between $\Delta_{F}$ and $\Omega_{b}^{X}$ has been described for boresight and non-boresight directions. From this procedure, the functions $\Delta_{F}\left(\Omega_{b}^{X}, M\right)$ and $\Delta_{F}\left(\Omega_{b}^{X}, \theta_{\max }, \theta_{\lim }\right)$ have been determined numerically and approximated by $\tilde{\Delta}_{F}\left(\Omega_{b}^{X}, M\right)$ and $\tilde{\Delta}_{F}\left(\Omega_{b}^{X}, \theta_{\max }, \theta_{\text {lim }}\right)$ in a systematic manner for $X=80 \%$. Consequently, the quadratic phase law $\phi_{m}$ can be determined systematically too for an aimed beam width $\Omega_{b}^{80}$ and steering angle $\theta_{\max }$. In this section, the accuracy of the proposed systematic beam broadening method is assessed by comparing the aimed beam width $\Omega_{b}^{80}$ with the effective one. This evaluation actually measures the accuracy of the approximation $\tilde{\Delta}_{F}$. The precision of the suggested beam broadening method is evaluated regarding the antenna array size $M$ for the boresight direction and regarding the steering

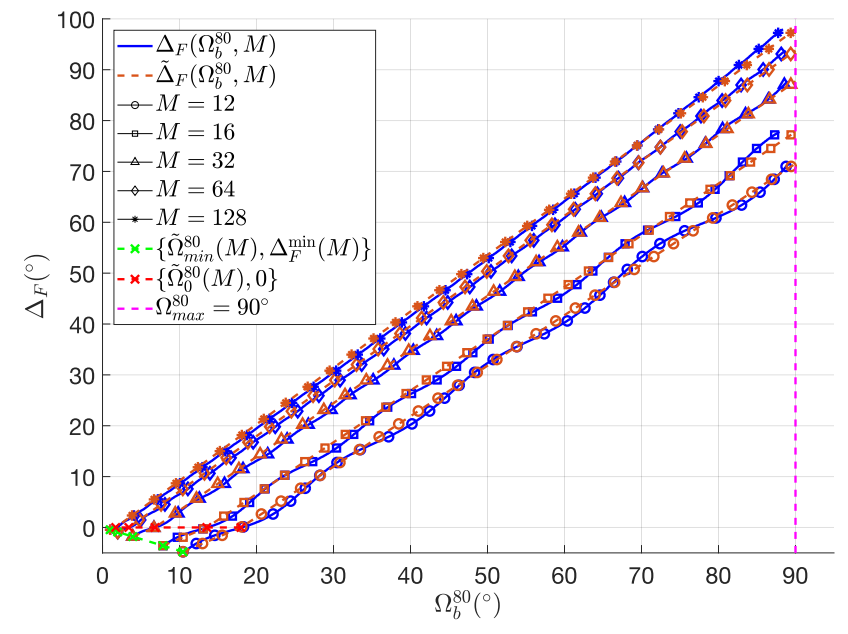

(a) $\Delta_{F} \in\left[-5^{\circ}, 100^{\circ}\right]$

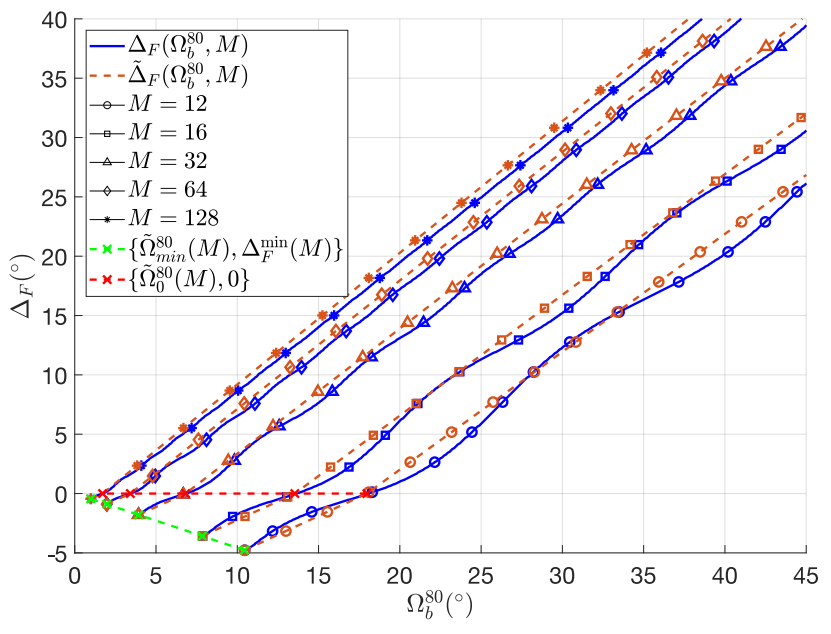

(b) $\Delta_{F} \in\left[-5^{\circ}, 40^{\circ}\right]$

Figure 10: $\Delta_{F}$ and $\tilde{\Delta}_{F}$ versus $\Omega_{b}^{80}$ for $M \in\{12,16,32,64,128\}$ 
angle $\theta_{\max }$ for non-boresight directions. In the latter case, the maximum drift between the desired steering angle $\theta_{\max }$ and the barycenter $\theta_{b}$ is also measured.

\section{A. Boresight direction}

The mean relative error and the mean absolute error between the aimed beam width $\Omega_{b}^{80}$ and the effective one are the metrics chosen for assessing the accuracy of the proposed systematic beam broadening method. The simulations are performed for $\Omega_{b}^{80} \in\left[\Omega_{\min }^{80}(M), \Omega_{\max }^{80}=90^{\circ}\right]$ and the obtained functions $\Delta_{F}\left(\Omega_{b}^{80}, M\right)$ and $\tilde{\Delta}_{F}\left(\Omega_{b}^{80}, M\right)$ are depicted in Figure 10 for $M \in\{12,16,32,64,128\}$. It is observed that those two functions are close to each other whatever the array size $M$, which is confirmed by the results obtained for the mean relative error and the mean absolute error (Figure 11). The approximations suggested in Section IV-C shouldn't lead to a mean relative/absolute error that exceeds $2.5 \% / 0.8^{\circ}$

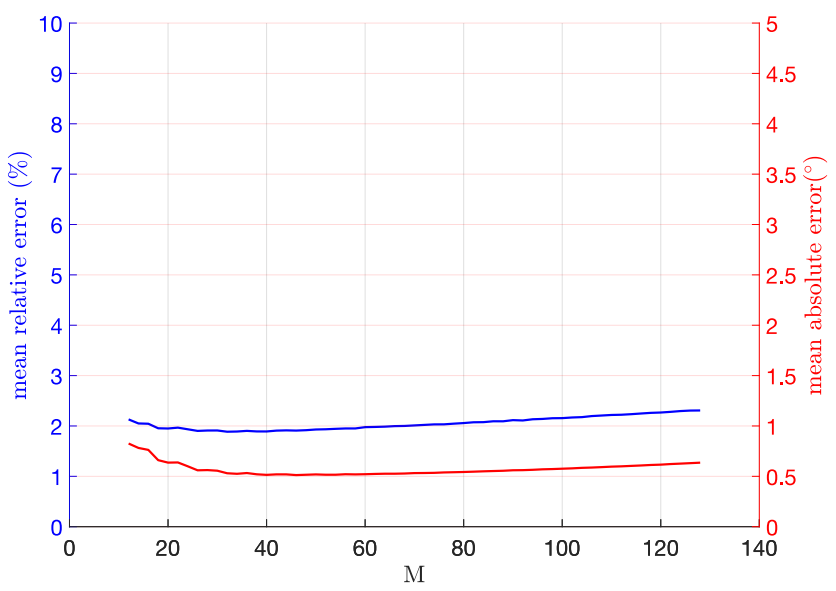

Figure 11: Mean relative and absolute error between the effective beam width and $\Omega_{b}^{80}$ for $\Omega_{b}^{80} \in\left[\Omega_{\min }^{X}(M), \Omega_{\max }^{X}\right]$ and $M \in[12,128]$.

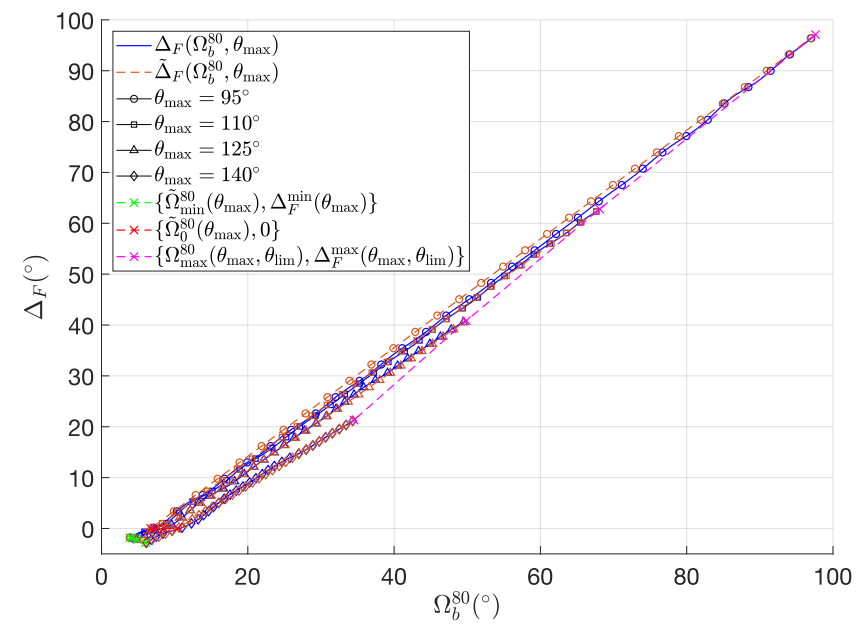

(a) $\Delta_{F} \in\left[-20^{\circ}, 100^{\circ}\right]$ for an antenna array size $M \in[12,128]$. Note that the mean absolute error is higher for small $M$ values since the relation between $\Delta_{F}\left(\Omega_{b}^{X}, M\right)$ and $\Omega_{b}^{X}$ is not strictly linear as observed in Figure $10 \mathrm{~b}$.

\section{B. Non-boresight directions}

The mean relative error between the aimed beam width $\Omega_{b}^{80}$ and the effective one is the metrics chosen for assessing the accuracy of the proposed systematic beam broadening method for non-boresight directions, considering an antenna array composed of $M=32$ antenna elements. In addition, the maximum drift between the desired steering angle $\theta_{\max }$ and the barycenter $\theta_{b}$ is calculated numerically to check that the $\theta_{\lim }$ constraint introduced in Section IV-D is verified. The simulations are performed for $\Omega_{b}^{80} \in\left[\Omega_{\min }^{80}\left(\theta_{\max }\right), \Omega_{\max }^{80}\left(\theta_{\max }, \theta_{\lim }=2.5^{\circ}\right)\right]$ and $\theta_{\max } \in$ $\left[95^{\circ}, 140^{\circ}\right]$ with an angular resolution of $5^{\circ}$. As the array factors obtained for a steering angle $\theta_{\max }=\theta_{0}$ and $\theta_{\max }=$ $\pi-\theta_{0}$ are symmetrical about $\theta=90^{\circ}$, the interval $\theta_{\max } \in$ $\left[40^{\circ}, 85^{\circ}\right]$ is not evaluated since the performance would be exactly the same than the one obtained for $\theta_{\max } \in\left[95^{\circ}, 140^{\circ}\right]$. The obtained functions $\Delta_{F}\left(\Omega_{b}^{80}, \theta_{\max }, \theta_{\lim }=2.5^{\circ}\right)$ and $\tilde{\Delta}_{F}\left(\Omega_{b}^{80}, \theta_{\max }, \theta_{\lim }=2.5^{\circ}\right)$ are depicted in Figure 12 for $\theta_{\max } \in\left\{95^{\circ}, 110^{\circ}, 125^{\circ}, 140^{\circ}\right\}$. As expected, it is observed that the width of $\Omega_{b}^{80}$ range decreases as $\theta_{\max }$ increases so as to limit the maximum drift to $\theta_{\lim }=2.5^{\circ}$. The red curve depicted in Figure 13 validates the approach described in Section IV-D since the maximum drift between the desired steering angle $\theta_{\max }$ and the barycenter $\theta_{b}$ equals $\theta_{\lim }$ whatever $\theta_{\text {max }}$. In addition, the mean relative error between the aimed beam width $\Omega_{b}^{80}$ and the effective one doesn't go beyond $3 \%$, which seems fairly accurate. Note that the lower precision of the suggested method for steering angles close to the boresight directions is attributable to the greater width of $\Omega_{b}^{80}$ range.

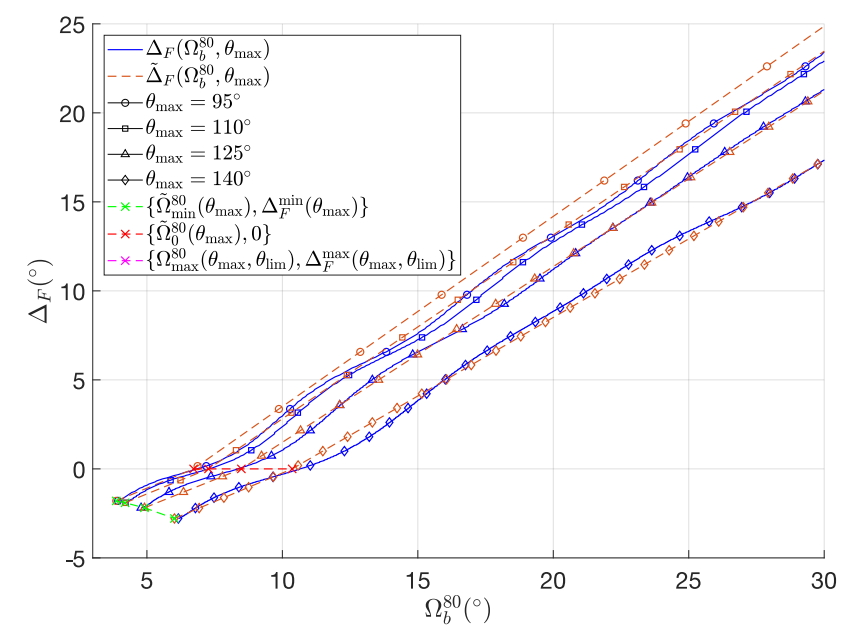

(b) $\Delta_{F} \in\left[-5^{\circ}, 25^{\circ}\right]$

Figure 12: $\Delta_{F}$ and $\tilde{\Delta}_{F}$ versus $\Omega_{b}^{80}$ for $M=32, \theta_{\lim }=2.5^{\circ}$ and $\theta_{\max } \in\left\{95^{\circ}, 110^{\circ}, 125^{\circ}, 140^{\circ}\right\}$ 


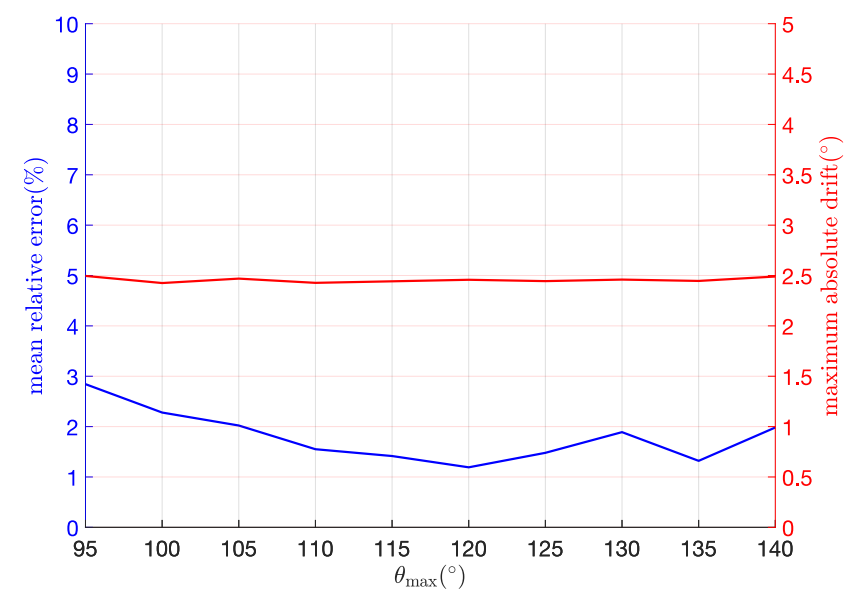

Figure 13: Mean relative error between the effective beam width and $\Omega_{b}^{80}$ for $\Omega_{b}^{80} \in$ $\left[\Omega_{\min }^{X}\left(\theta_{\max }\right), \Omega_{\max }^{X}\left(\theta_{\max }, \theta_{\lim }=2.5^{\circ}\right)\right]$ and $\theta_{\max } \in$ $\left[95^{\circ}, 140^{\circ}\right]$. The maximum absolute drift between the steering angle $\theta_{\max }$ and the barycenter $\theta_{b}$ is evaluated on the same intervals.

\section{CONCLUSION}

In this paper, the far-field radiation pattern produced by a continuous line-source for a quadratic phase excitation has been derived. An intuitive explanation of the beam broadening phenomenon, that involves the Fresnel functions, has also been provided for a better understanding of the quite complex expression that characterized the space factor for that type of excitation. It has also been shown that the quadratic coefficient of the phase law can be expressed as a function of the angular distance between the cosine Fresnel functions. As discussed in the paper, this angular distance precisely lead the beam width for very large antenna arrays but not for common size ones. Consequently, a procedure that enables to tune systematically the quadratic coefficient according to the desired beam width has been designed for boresight and non-boresight directions. In addition to being systematic, the proposed solution provides an accurate beam width control as shown by the simulation results. From the perspective of authors, the solution detailed in this paper has the benefit of being easily implementable considering both complexity aspect and hardware constraints, making it well-suited for mobile wireless communications in the millimeter band.

Further improvements could still be envisioned. Indeed, the current procedure enable to determine the quadratic coefficient whatever the array size for the boresight direction and whatever the steering angle for non-boresight directions. The next step would be to generalize the approach whatever the array size and the steering angle for non-boresight directions. Moreover, it would be interesting to extend the concept to planar arrays as 3D-beamforming is a key concept for the new generation of mobile communication systems.

\section{DECLARATIONS}

\section{A. Availability of data and materials}

Not applicable

\section{B. Competing Interest}

The authors declare that they have no competing interests.

\section{Funding}

This work has been supported by the Orange company.

\section{Authors contribution}

CF came up with the initial idea of beam broadening control by means of Fresnel functions, completed the analytical derivations, and conducted the simulations. MC proposed to characterize the beam width from the beam efficiency of the array. BJ suggested the barycenter metrics for non-boresight directions. $\mathrm{CF}$ and $\mathrm{MC}$ wrote this paper. All authors read and approved the final manuscript.

\section{E. Acknowledgements}

Not applicable

\section{F. Authors Information}

Corentin Fonteneau received M.S. degree in electrical engineering from INSA Rennes, Rennes, France, in 2018. $\mathrm{He}$ is currently pursuing the Ph.D degree in Orange Labs, Rennes. His current research interests include massive MIMO, millimeter wave communication, ultra reliable and low latency communication.

Matthieu Crussière received the M.S. and Ph.D. degrees in electrical engineering from the National Institute of Applied Sciences, France, in 2002 and 2005, respectively. Since 2005 , he has been an Associate Professor affiliated to the Research Institute of Electronics and Telecommunications, Rennes, France. In 2014, he started collaborations as an Associate Researcher with the Institute of Research and Technology B-COM, Rennes. He has authored or co-authored over 80 technical papers in international conferences and journals. He has been involved in several European and French national research projects in the field of powerline, broadcasting, ultrawideband, and mobile radio communications. His main research interests lie in digital communications and signal processing, with a particular focus on multicarrier and multiantenna systems.

Bruno Jahan received the M.S. degree in optical and photonics and the M.S. degree in electronic systems from the University of Paris-Sud, Orsay, France, in 1989 and 1990, respectively. In 1991, he was with Télédiffusion de France as a Research Engineer. He joined Orange Labs (formerly France Telecom), Rennes, in 1998. His research interests include digital signals processing for wire and wireless communications. 


\section{REFERENCES}

[1] C. Anderson and T. Rappaport, "In-building wideband partition loss measurements at 2.5 and 60 ghz," IEEE Transactions on Wireless Communications, vol. 3, no. 3, pp. 922-928, 2004.

[2] K. C. Allen, "Building penetration loss measurements at $900 \mathrm{mhz}, 11.4$ ghz, and $28.8 \mathrm{ghz}, " 1994$.

[3] G. R. MacCartney, S. Deng, S. Sun, and T. S. Rappaport, "Millimeterwave human blockage at $73 \mathrm{ghz}$ with a simple double knife-edge diffraction model and extension for directional antennas," in 2016 IEEE 84th Vehicular Technology Conference (VTC-Fall), 2016, pp. 1-6.

[4] S. Orfanidis, Electromagnetic Waves and Antennas.

[5] M. Cheng et. al., "Coverage analysis for millimeter wave cellular networks with imperfect beam alignment," IEEE Trans. Veh. Technol., vol. 67, no. 9, pp. 8302-8314, 2018.

[6] M. Giordani et. al., "A tutorial on beam management for 3GPP-NR at mmwave frequencies," Commun. Surveys Tuts., vol. 21, no. 1, pp. 173-196, 2019.

[7] J. E.Dahlman, S.Parkvall, $5 G N R$ : The next generation wireless access technology,. New York, NY, USA: Academic Press, 2018.

[8] S. Rajagopal, S. Abu-Surra, and M. Malmirchegini, "Channel feasibility for outdoor non-line-of-sight mmwave mobile communication," in 2012 IEEE Vehicular Technology Conference (VTC Fall), 2012, pp. 1-6.

[9] M. Robaei and R. Akl, "Millimeter-wave blockage modeling and mitigation," in 2021 IEEE International Workshop Technical Committee on Communications Quality and Reliability (CQR 2021), 2021, pp. 1-6.

[10] E. Bayliss, "A phase synthesis technique with application to array beam broadening," in 1966 Antennas and Propagation Society International Symposium, vol. 4, 1966, pp. 427-432.

[11] M. Mofrad, Reza Fatemi et. al., "Comparison of antenna beam broadening methods for phased array radar applications," in 2011 Loughborough Antennas Propagation Conference, 2011, pp. 1-4.

[12] C. A. Balanis, Antenna theory: analysis and design. Wiley-Interscience, 2005.

[13] M. Heath, Robert W.et. al., "An overview of signal processing techniques for millimeter wave mimo systems," IEEE Journal of Selected Topics in Signal Processing, vol. 10, no. 3, pp. 436-453, 2016.
[14] J. A. Zhang, X. Huang, V. Dyadyuk, and Y. J. Guo, "Massive hybrid antenna array for millimeter-wave cellular communications," IEEE Wireless Communications, vol. 22, no. 1, pp. 79-87, 2015.

[15] A. Molish et. al., "Hybrid beamforming for massive mimo: A survey," IEEE Commun. Mag., vol. 55, no. 9, pp. 134-141, 2017.

[16] Z. Pi and F. Khan, "An introduction to millimeter-wave mobile broadband systems," IEEE Communications Magazine, vol. 49, no. 6, pp. 101-107, 2011.

[17] S. Rajagopal, "Beam broadening for phased antenna arrays using multibeam subarrays," in 2012 IEEE International Conference on Communications (ICC), 2012, pp. 3637-3642.

[18] H. Lebret and S. Boyd, "Antenna array pattern synthesis via convex optimization," IEEE Transactions on Signal Processing, vol. 45, no. 3 pp. 526-532, 1997.

[19] A. Elshafiy and A. Sampath, "Beam broadening for $5 \mathrm{~g}$ millimeter wave systems," in 2019 IEEE Wireless Communications and Networking Conference (WCNC), 2019, pp. 1-6.

[20] V. Raghavan, J. Cezanne, S. Subramanian, A. Sampath, and O. Koymen, "Beamforming tradeoffs for initial ue discovery in millimeter-wave mimo systems," IEEE Journal of Selected Topics in Signal Processing, vol. 10, no. 3, pp. 543-559, 2016.

[21] K. Sayidmarie and Q. Sultan, "Synthesis of wide beam array patterns using quadratic-phase excitations," International Journal of Electromagnetics and Applications, vol. 3, pp. 127-135, 122013.

[22] J. R. Klauder, A. C. Price, S. Darlington, and W. J. Albersheim, "The theory and design of chirp radars," The Bell System Technical Journal, vol. 39, no. 4, pp. 745-808, 1960.

[23] M. Abramowitz and I. A. Stegun, Handbook of Mathematical Functions with Formulas, Graphs, and Mathematical Tables, ninth dover printing, tenth gpo printing ed. New York: Dover, 1964

[24] C. Fonteneau, M. Crussière, and B. Jahan, "A systematic beam broadening method for large phased arrays," in 2021 Joint European Conference on Networks and Communications 6G Summit (EuCNC/6G Summit), 2021, pp. 7-12. 